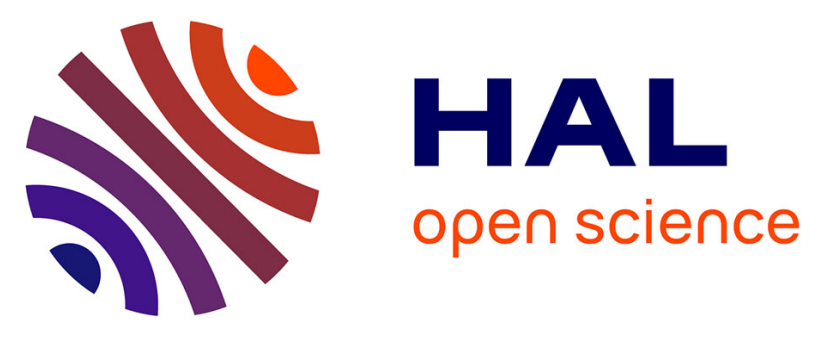

\title{
Impact of peripheral fragmentation on the steam gasification of an isolated wood charcoal particle in a diffusion-controlled regime
}

Fabrice Golfier, Laurent van de Steene, Sylvain Salvador, Floriane Mermoud, Constantin Oltean, Michel Bues

\section{To cite this version:}

Fabrice Golfier, Laurent van de Steene, Sylvain Salvador, Floriane Mermoud, Constantin Oltean, et al.. Impact of peripheral fragmentation on the steam gasification of an isolated wood charcoal particle in a diffusion-controlled regime. Fuel, 2009, 88 (8), p.1498-1503. 10.1016/j.fuel.2009.02.043 . hal-01846916

\section{HAL Id: hal-01846916 https://hal.science/hal-01846916}

Submitted on 6 Nov 2018

HAL is a multi-disciplinary open access archive for the deposit and dissemination of scientific research documents, whether they are published or not. The documents may come from teaching and research institutions in France or abroad, or from public or private research centers.
L'archive ouverte pluridisciplinaire HAL, est destinée au dépôt et à la diffusion de documents scientifiques de niveau recherche, publiés ou non, émanant des établissements d'enseignement et de recherche français ou étrangers, des laboratoires publics ou privés. 


\title{
Impact of peripheral fragmentation on the steam gasification of an isolated wood charcoal particle in a diffusion-controlled regime
}

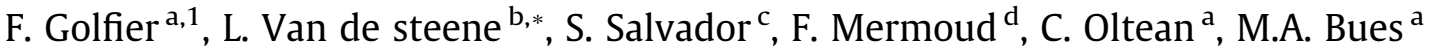 \\ a Laboratoire Environnement Géomécanique et Ouvrages (LAEGO), Nancy Université, Rue du Doyen Roubault BP 40, F-54501 Vandoeuvre-lès-Nancy, France \\ ${ }^{\mathrm{b}}$ CIRAD-Forêt, UPR 42 Biomasse Energie, TA 10/16, 73 rue JF Breton, 34398 Montpellier Cedex 5, France \\ ${ }^{c}$ RAPSODEE, UMR 2392 CNRS, Ecole des Mines d'Albi-Carmaux, Campus Jarlard, 81013 Albi Cedex 09, France \\ ${ }^{\mathrm{d}}$ University of Geneva, Energy Group, Battelle Bat. D, Route de Drize 7, CH-1227 Carouge, Switzerland
}

\section{A B S T R A C T}

This study focuses on the effect of peripheral fragmentation during gasification of an isolated wood charcoal particle in the regime of diffusional limitations. In fact, all the models available in the literature fail to reproduce the specific changes in biomass charcoals for which conversion increases quasi linearly versus time, whatever the particle size. We assumed this discrepancy is partly due to the fragmentation phenomenon, i.e. the detachment of fragments from the surface of the particle, which is not usually taken into account in models. The classical assumption in percolation models is a critical value of porosity beyond which fragmentation occurs. We compared our model results with experimental data. We demonstrated that in the diffusion-limited regime, the direct extrapolation of such a method to a continuum model is not satisfying. A new criterion of fragmentation based on a critical porosity and a critical porosity gradient is proposed which considerably improves modelling of gasification charcoal particles.

Keywords:

Biomass

Gasification

Fragmentation

Charcoal

Particle

\section{Introduction}

Gasification has emerged as a clean and effective way of producing gas from biomass, particularly from wood. The main transformation in such processes is the reaction between the charcoal -or char- (carbonaceous solid residue after wood pyrolysis) and steam. This heterogeneous $\mathrm{C}-\mathrm{H}_{2} \mathrm{O}$ reaction produces the majority of $\mathrm{H}_{2}$ and $\mathrm{CO}$ that can be valorised either for electricity or for the production of biofuels. In fixed bed gasifiers, this endothermic reaction occurs at temperatures in the range of $800-1200{ }^{\circ} \mathrm{C}$ and requires $\mathrm{H}_{2} \mathrm{O}$ and a heat supply inside the bed. Regarding the size of the particles (around 1-2 cm), limitations due to mass and heat transfers cannot be ignored. Basically, the reaction can be described as follows:

$\mathrm{C}+\mathrm{H}_{2} \mathrm{O} \rightarrow \mathrm{CO}+\mathrm{H}_{2}$

Given the lack of knowledge about the charcoal gasification process, a single particle gasified under steam has frequently been studied both experimentally and numerically.

As a result, several numerical models have been developed at the single particle scale [1-6]. Although these models enable a better understanding of all the highly coupled phenomena involved at the particle scale, in the case of biomass charcoals, none has

\footnotetext{
* Corresponding author. Tel.: +33467616522.

E-mail addresses: fabrice.golfier@ensg.inpl-nancy.fr (F. Golfier), steene@cirad.fr (L. Van de steene).

${ }^{1}$ Tel.: +33 (0) 3835963 44; fax: +33 (0) 383596300 .
}

succeeded in satisfactorily matching experimental results beyond a critical value of conversion. The term "conversion" is used in this study to refer to the macroscopic average conversion, i.e. the ratio of the total mass lost - by gasification or fragmentation - to the initial mass of the particle. Indeed, this kind of char presents a conversion level $X$ which increases quasi-linearly versus time, in spite of diffusional effects. As emphasized by Struis et al. [7] and Mermoud et al. [6], models tend to overestimate the influence of internal transfers for large particles and hence fail to reproduce this linear evolution satisfactorily. In Dasappa et al. [1], for example, the comparison between predictions and experiments was valid only up to a conversion level of $60 \%$. A similar comment can be made about the simulations conducted by Mermoud et al. [6].

There are several possible explanations for this discrepancy. Firstly, it should be recalled that all the models available in the literature are spherically symmetric one-dimensional models, based on the assumption of an isotropic material. But, as the wood is initially anisotropic, the charcoal loses its initial shape during gasification and shrinks, preferentially in a transversal direction. This shorter dimension facilitates mass and heat transfers inside the particle in comparison with the predictions of the model. As a consequence, although this assumption is relevant at low conversion levels, i.e. before the particle size decreases, at the end of gasification process it leads to overestimation of the conversion rate $d X / d t$.

Secondly, several authors [5-10] have suggested that such behaviour might result from peripheral fragmentation (also called secondary fragmentation), i.e. the disintegration of the porous 


$\begin{array}{ll}\text { Nomenclature } \\ d & \text { charcoal particle diameter }(\mathrm{mm}) \\ p^{*} & \text { probability of site occupation }(-) \\ p^{*} & \text { percolation threshold }(-) \\ t & \text { time }(\mathrm{s}) \\ X & \text { conversion level }(-)\end{array}$

charcoal into small fragments at the particle surface. Note that a similar process can be observed with coal chars [14-19], even if the fragmentation mode may differ between both chars due to the difference of structure (e.g., variation of volatile-matter content or of the initial porosity). The above-mentioned phenomenon can be considered as a percolative fragmentation process [11-13]. Indeed, under the control of external diffusion, fragmentation can enhance the conversion rate. In these conditions, the gasification process involves the disappearance of the solid phase and the formation of a porosity gradient due to the mass transfer effects, until the local porosity of the superficial slice of the particle reaches a critical value. At this point, the charcoal structure disintegrates at the surface and the radius of the particles decreases. The physical result is that when too much carbon has been removed from the charcoal structure, the remaining matrix can no longer maintain its structure and a fragment detaches from the particle. The existence of this process was first confirmed experimentally by Kerstein and Niksa [14] and Sundback et al. [15] for coal chars. Mitchell and Akanetuk [16] suggested that fragmentation can reduce the initial particle mass by around $20-30 \%$. Zhang et al. [17] studied the influence of operating conditions on the fragmentation of coal particles in a fluidized bed. Feng and Bhatia [18] contributed to the understanding of the phenomenon by measuring the variation in electrical resistivity with conversion and by direct observation, of the fragments. Their results imply, in particular, that peripheral fragmentation occurs at early stages of gasification with low conversion levels and is conditioned by diffusive effects. Baxter [19] also reported a strong influence of particle size on the extent of fragmentation. It should be noted that, even in the kinetically controlled regime, fragmentation can induce a variation in the conversion rate, due to the exposure of a fresh surface area, and hence an increase in reactive area, accompanying the particle gasification process $[7,20]$.

Many researchers have studied the fragmentation phenomenon in both particle models [5,9,20-24] and in reactors models, laminar flow reactors [25] or fluidized beds [26,27]. Such models can be classified in three distinct approaches:

- A discrete or network approach [9,21,23,24,28,29] where the single char particle is seen as a two or three-dimensional lattice composed of a large number of small clusters (cf. Sahimi et al. [13] for a review of these models). Results are interesting from a qualitative point of view but require previous knowledge of the char microstructure.

- A stochastic or statistical approach [14,30-33] based on the Monte Carlo method. The fragmentation process is described by different statistical functions such as the number of fragments generated, which need to be fitted.

- A continuum approach $[5,16,25,34]$ based on percolation theory, where particle fragmentation occurs when porosity exceeds a critical value $\varepsilon^{*}$. The relative simplicity of this method means it can easily be incorporated in gasification continuum models. However, few studies included quantitative comparison with experimental results, and still fewer for particles with a radius of more than a few millimetres.
This study focuses on the latter formulation and attempts to explain the discrepancy observed at high conversion levels between experimental and numerical results by taking the fragmentation phenomenon into account in a particle gasification model. In order to validate our assumptions, we used the Mermoud et al. [6] model. First, we show that the classical percolative approach is not really suitable to predict char gasification when there are significant limitations due to mass and heat transfer within the particle. Second, we propose a new formulation to characterize the peripheral percolation phenomenon and compare it with experimental results obtained by Mermoud et al. [6].

\section{One-dimensional gasification modelling at the scale of one particle}

In this section, we first briefly recall the numerical model developed by Mermoud et al. [6] for steam gasification of a single charcoal particle and the main results obtained. This model, based on balance equations for heat and gas species and including heterogeneous reaction kinetics, is derived under a spherically symmetric one-dimensional form. A Langmuir-Hinshelwood formulation that accounts for hydrogen inhibition is used for the steam gasification kinetics.

As for the equation of state, the ideal gas law is applied. No simplifying assumption is adopted concerning the evolution of the charcoal particle during gasification. Thus, the description of the transport of the products in conjunction with the increase in porosity covers the entire range from the limiting case of a reacting-core model (reaction-rate limited regime) to a shrinking core model (mass-transfer limited regime).

The boundary conditions are obtained by considering the heat and mass fluxes (including radiative transfer) at the charcoal/ atmosphere interface.

At each time step, once the pressure and velocity fields are determined, temperature and concentration fields can be calculated for the different species. At this point, the new properties of the medium (particle diameter, porosity, permeability, effective diffusion and conductivity) can be updated. In particular, local porosity varies during gasification as a function of the reaction term, i.e. the quantity of solid carbon consumed by the chemical reaction. In the current version of the model - in which fragmentation is not included - when the porosity of the cell considered reaches 1 ( 0.999 for numerical purposes), this cell is removed and the particle shrinks.

Comparison with experimental data indicated that the model predictions are both qualitatively and quantitatively satisfactory, despite the fact that the phenomena of reactive surface variation and particle fracturing are not well understood.

Indeed, during experiments under all conditions, conversion follows an almost linear slope with time. Fig. 1 shows the typical evolution of the conversion as a function of time for different particle sizes (7, 14 and $21 \mathrm{~mm}$ diameter). As can be seen in the figure, the model can predict the slope in the case of small particles where the reaction kinetics is dominant, but cannot show the conversion 


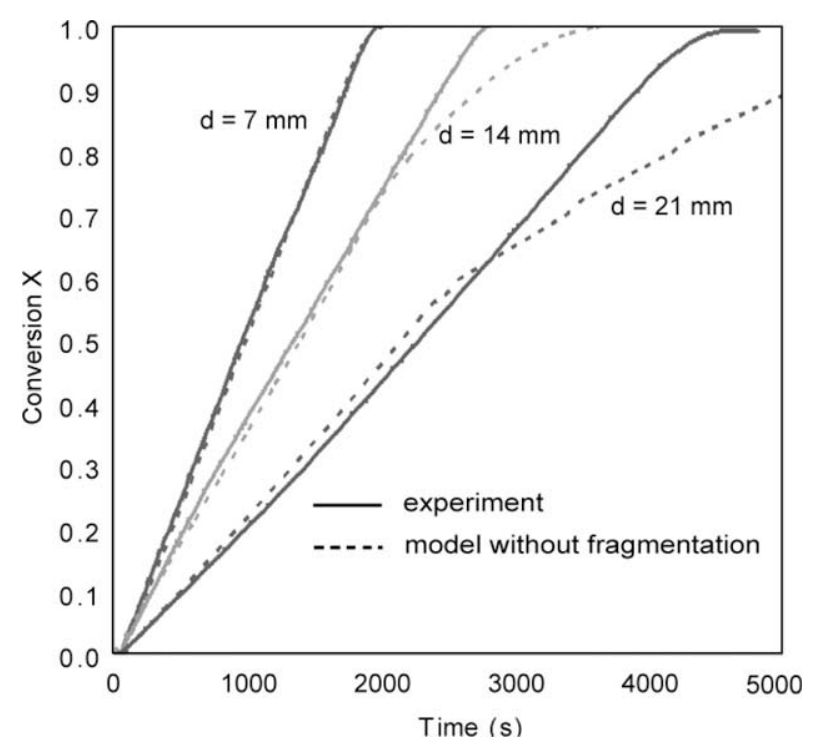

Fig. 1. Influence of particle diameter on changes in the conversion rate $\left(T=930^{\circ} \mathrm{C}\right.$, $P_{\mathrm{H} 2 \mathrm{O}}=0.2 \mathrm{~atm}$ ) (from Ref. [6]).

of larger particles beyond roughly $60 \%$ conversion. As mentioned above, this relative lack of accuracy beyond a critical value of conversion is not specific to this model but its origin still remains problematic. We suspect that this discrepancy is due to the fact that the model does not take into account the particle fragmentation phenomenon that occurs during gasification.

In the following section, we examine the peripheral fragmentation process and determine which criterion of fragmentation is the most convenient.

\section{Peripheral fragmentation: which is the most convenient criterion?}

Peripheral fragmentation is usually related to the percolation phenomenon [35-37]. Basically, a porous medium can be seen as a square lattice, i.e. an infinite array of squares. All of them correspond to a site which can be either occupied or vacant, depending on whether it represents solid material or a pore. The sites are assumed to be occupied randomly with a probability $p$, which depends on porosity. When two adjacent sites are occupied, they are said to be connected. Several sites that are interconnected form clusters. Thus, the whole porous matrix constitutes an infinite cluster.

Carbon is consumed throughout the gasification reaction, and as a result, some sites are removed. If a particle remains at constant size - no compaction or shrinking - the disappearance of sites leads to an increase in porosity, and hence a decrease in probability $p$. As we will see later, such a transition usually suggests a major transformation in the infinite cluster. For instance, one could ask what is the smallest probability at which the porous material can maintain its structure.

In the kinetic regime, percolation theory predicts that a low enough probability, or high enough porosity, will cause the particle to disintegrate into fragments. This critical probability $p^{*}$ or critical porosity $\varepsilon^{*}$ at which the porous matrix can no longer maintain its structure is called percolation threshold. In the kinetic regime, this condition is reached simultaneously throughout the particle, whereas in the diffusion-controlled regime, a porosity gradient appears leading to the formation of a fragmentation front. Indeed, under the action of the chemical reaction, connections that link a group of neighbouring occupied sites to the general cluster can be broken and cause part of the porous material to detach: this is the peripheral fragmentation phenomenon.
Several authors $[5,16,25,34]$ have used this approach to include the fragmentation process in their gasification continuum model. At each node, porosity increases over time proportionally to the amount of carbon consumed until a certain critical value $\varepsilon^{*}$ is reached when the total solid mass suddenly disintegrates by disaggregation. The above value of $\varepsilon^{*}$ takes into account only topological characteristics of the char microstructure leading to particle breakup [9]. Hence, it is assumed to be constant, regardless of particle size. Although this approach is convenient in the kinetic regime, it is problematic in the diffusion-limited regime.

Fig. 2 illustrates the coupling of this method to the numerical model developed by Mermoud et al. [6]. Two different values of $\varepsilon^{*}$ are used here, 0.9 and 0.999 (i.e. no fragmentation occurs). Simulations are performed with both criteria and two different particle sizes ( 7 and $21 \mathrm{~mm}$ diameter), and compared with experimental data. Temperature and steam partial pressure are constant and fixed respectively, at $930^{\circ} \mathrm{C}$ and 0.2 atm. Fig. 2 shows that, as explained in the previous section, without fragmentation, the model satisfactorily reproduces the behaviour of a small gasifying particle for which the reaction kinetics is dominant, but internal diffusive limitations are overestimated when the particle size increases. Conversely, for a critical porosity value equal to 0.9 , the model successfully predicts the gasification of the $21 \mathrm{~mm}$ diameter particle but fails to reproduce the slope for the smallest one.

Indeed, several experimental studies [18-19,33] confirmed that the extent of fragmentation depends to a great extent on mass and heat transfer limitations, which can vary as a function of the operating conditions such as particle size and temperature. In particular, it has been observed that peripheral fragmentation may start at a value of $p$ higher than the percolation threshold. As the reaction progresses, porosity increases locally -especially at the surfaceunder the effect of diffusive gradients. Thus, some fragments are isolated from the core of the particle, i.e. the infinite cluster, and detach from the surface, removing the carbon at this location. Nevertheless, the remaining matrix maintains its structure and does not collapse until the percolation threshold is reached. If the above-mentioned approach can capture such a process, it predicts a mass of detached fragments directly proportional to the mass of the initial char particle. But the percolation theory [37] predicts that the distribution of fragments varies linearly with particle mass in a log-log plot. Experimental measurements by Kerstein and Niksa [14] and Feng and Bhatia [18] support this observation.

Simulations by Sahimi and Tsotsis [29] also provided an interesting insight into this problem. In their model, based on a network

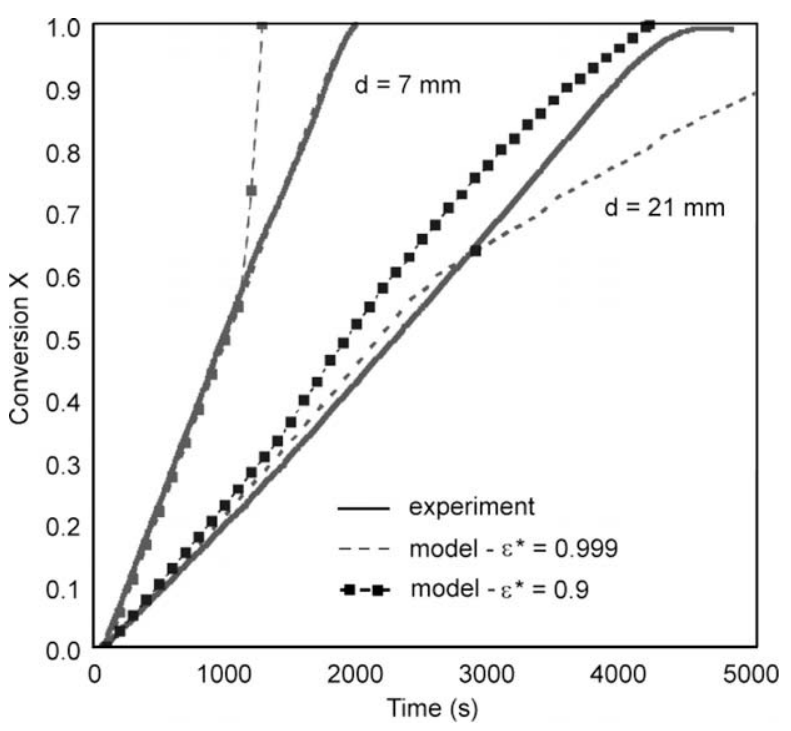

Fig. 2. Impact of the critical porosity criterion on simulations. 
approach, each occupied site can react with a probability $r$ representing the reactivity of the solid matrix. If $r \simeq 1$ (limit of the diffusion regime or shrinking core model), only the surface of the initial matrix is consumed and no major fragmentation of the particle takes place. This is the phenomenon of peripheral fragmentation. Conversely, if $r \simeq 0$ (kinetic regime or uniform particle model), the reactant penetrates deeply inside the network and matrix fragmentation can occur when the percolation threshold is reached. Between these limits, a porosity gradient appears within the particle (from a macroscopic point of view) and many fragments with a wide variety of shapes and sizes are produced as a function of the relative importance of kinetic and diffusional limitations. These are the operating conditions on which we focus.

From this discussion, it is obvious that the classical continuum approach based on a single critical value of porosity does not allow the peripheral fragmentation phenomenon to be satisfactorily taken into account. The underlying idea is that for the same value of probability $p$ (and hence porosity $\varepsilon$ ), several more or less stable configurations (with a different number of connections) are possible. As a consequence, a criterion of the percolation threshold, based only on porosity which is a macroscopic property, cannot describe such a phenomenon, and an additional criterion is required. Fig. 3 shows two different configurations of percolation sites. Both clusters lead to the same value of porosity, calculated on the entire domain represented (same number of occupied sites) but do not present the same stability from the point of view of the number of connections. For instance, let us consider that site $A$ of configuration 1 is consumed. This does not modify the global structure of the percolation cluster; porosity increases but fragmentation does not occur. Conversely, in configuration 2 , if site $B$ is consumed, the connection between the smaller cluster composed of three adjacent cells and the base, i.e. the charcoal particle, is broken, and part of the porous material detaches. Feng and Bhatia [18] suggest considering isolated clusters in the formation of fragments at the surface in addition to the percolation threshold. Based on this observation, we propose a new conceptual model where the particle is seen as an ensemble of interconnected isolated clusters, themselves made up of several occupied sites. From a macroscopic point of view, the occurrence of breakage of the remaining connections between isolated clusters and the matrix depends on stresses undergone by the particle, such as those induced by the weight of the char itself. The greater the diffusional effects exerted within the char, the more fragile the particle structure at the surface. Therefore, we suggest using the porosity gradient $\nabla \varepsilon^{c}$ as the criterion of particle structure weakness. Under this assumption, the fragmentation phenomenon occurs if $\varepsilon \geqslant \varepsilon^{*}$ (uniform percolation threshold) or if $\varepsilon \geqslant \varepsilon^{c}$ and $\nabla \varepsilon \geqslant \nabla \varepsilon^{c}$ (peripheral percolation threshold) with $\varepsilon^{c} \leqslant \varepsilon^{*}$. If particle gasification corresponds to a shrinking core or a uniform particle model, no porosity gradient appears and the classical criterion of critical porosity controls the fragmentation process. In intermediate conditions, a porosity gradient occurs, and an additional criterion of fragmentation is required.

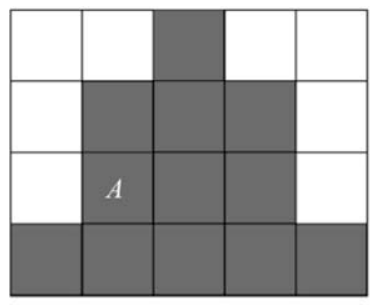

Configuration I

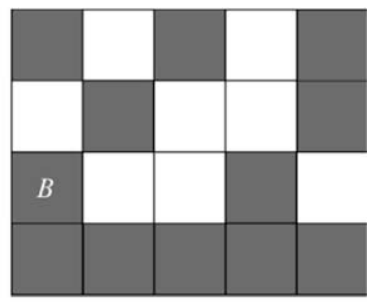

Configuration 2
Fig. 3. Two different configurations of clusters with the same probability $p$.
Below, by comparing our model with experimental results we demonstrate that such a criterion is valid and depends only on the intrinsic properties of the porous material.

\section{Results and discussion}

As explained above, the fragmentation process introduced in the model of Mermoud et al. [6] is now modified to start as soon as a critical value of the porosity and of the porosity gradient is reached. Simulation results are presented in Fig. 4 for three particle sizes, of which the operating conditions are similar to those detailed in Fig. 1. The results of the simulation are compared both to experiments and to the model without fragmentation. $\varepsilon^{c}$ and $\nabla \varepsilon^{\mathrm{c}}$ is fixed here at 0.92 and $18 \mathrm{~m}^{-1}$, respectively, from the observation of simulations. The percolation threshold $\varepsilon^{*}$ is kept at 0.999 . In this way, the same values of critical porosity and critical porosity gradient are used for all the simulations. Both parameters depend only on the intrinsic properties of the char under consideration and can consequently be modified if a different charcoal particle (with a different initial porosity for instance) is studied.

When reaction kinetics is the predominant mechanism (for the $7 \mathrm{~mm}$ diameter particle), the agreement obtained with the experimental curve remains excellent with or without fragmentation. On the other hand, when the particle is larger and the diffusional effects increase, the model including fragmentation is much more accurate.

Actually, a small particle is gasified practically uniformly without core shrinking. Porosity increases in a quasi homogeneous way and the porosity gradient consequently always remains lower than the fixed critical value. The char is completely gasified and no fragmentation occurs. Conversely, in the case of larger particles, the structure does not evolve uniformly and a porosity gradient quickly appears. Because of diffusional effects, porosity at the surface increases faster than inside the core. As soon as the two criteria (critical porosity and critical porosity gradient) are fulfilled, fragmentation occurs. Thus, as early as $30-40 \%$ of average conversion, the model predicts fragments will detach from the surface of the charcoal.

The ratio of mass lost by fragmentation and truly gasified mass for particles of 14 and $21 \mathrm{~mm}$ diameter is shown in Figs. 5 and 6, respectively. The fragmentation effect is negligible for the smallest

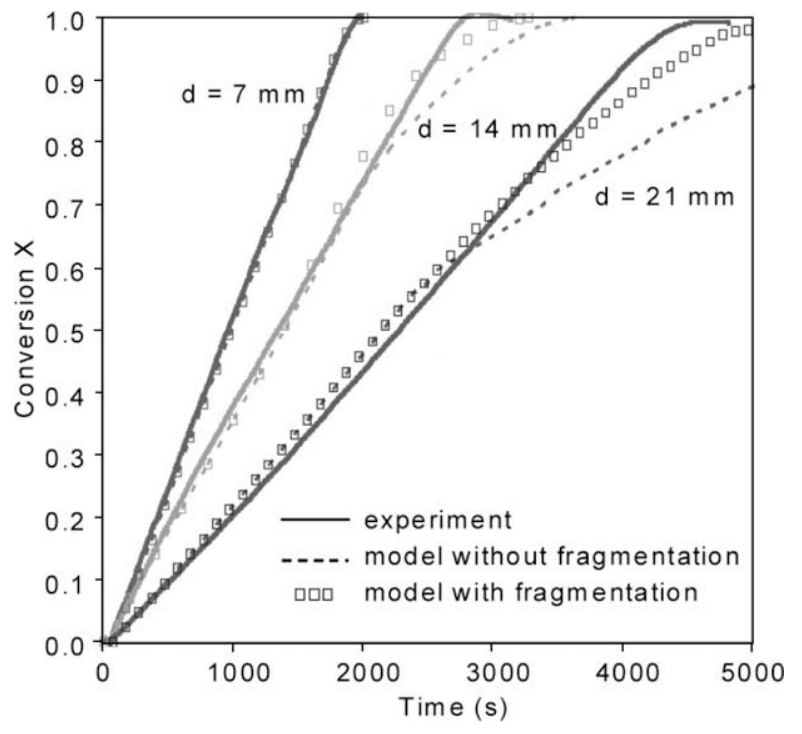

Fig. 4. Influence of particle diameter on changes in the conversion rate $\left(T=930^{\circ} \mathrm{C}\right.$, $\left.P_{\mathrm{H} 2 \mathrm{O}}=0.2 \mathrm{~atm}\right)-$ comparison between numerical simulations with or without fragmentation and experiments. 


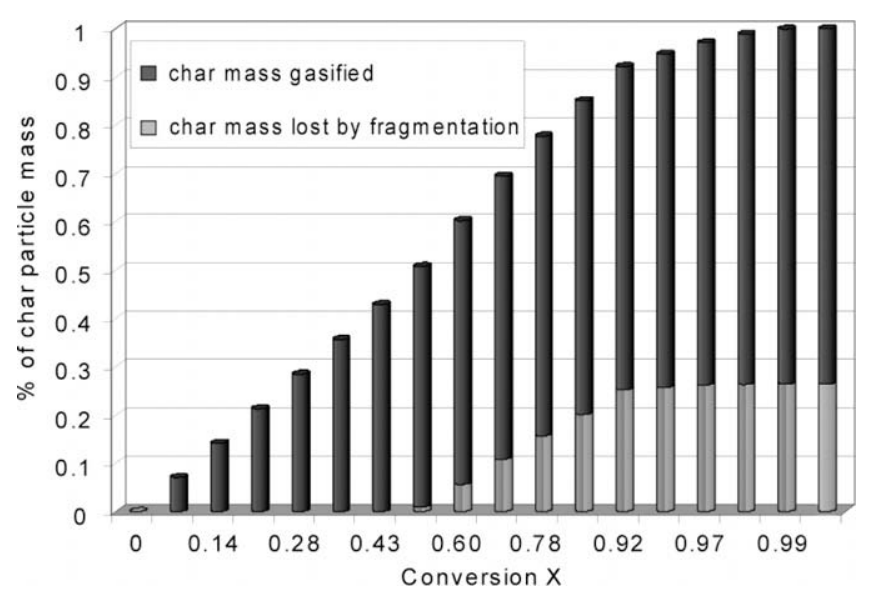

Fig. 5. Ratio of mass lost by fragmentation and truly gasified for a $14 \mathrm{~mm}$ diameter particle $\left(T=930^{\circ} \mathrm{C}, P_{\mathrm{H} 2 \mathrm{O}}=0.2 \mathrm{~atm}\right)$.

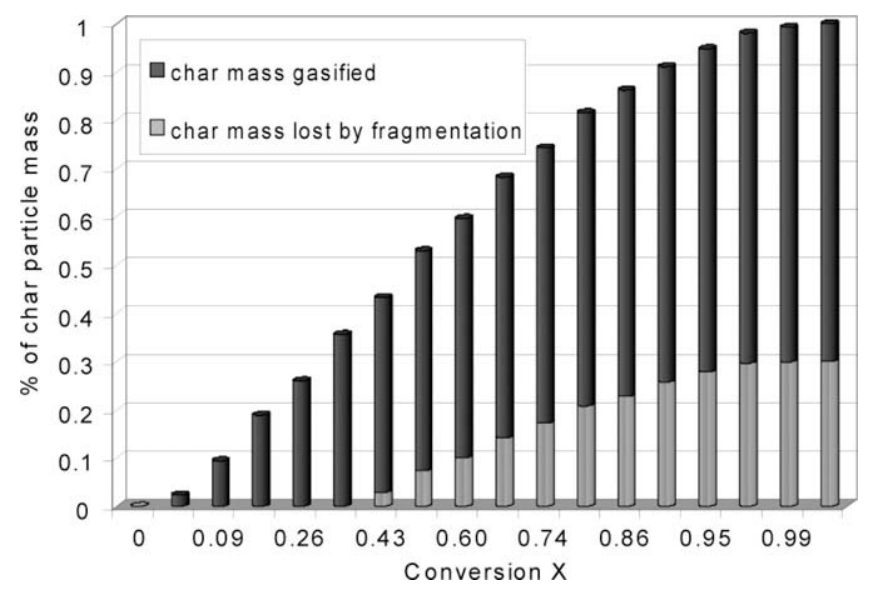

Fig. 6. Ratio of mass lost by fragmentation and truly gasified for a $21 \mathrm{~mm}$ diameter particle $\left(T=930^{\circ} \mathrm{C}, P_{\mathrm{H} 2 \mathrm{O}}=0.2 \mathrm{~atm}\right)$.

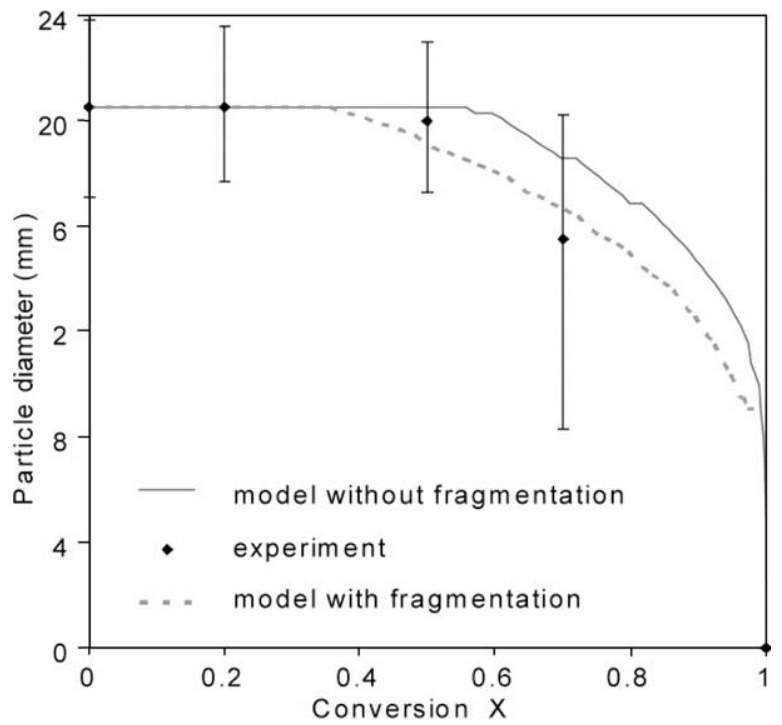

Fig. 7. Macroscopic evolution of a gasifying particle versus conversion $(0 \%, 20 \%, 50 \%$ and $70 \%$ conversion) and comparison with model predictions with or without fragmentation.

particle of $7 \mathrm{~mm}$ diameter. As expected, the fragmentation process starts earlier with the largest particle and has a greater impact (30\% instead of $26 \%$ of mass lost by fragmentation).
Another interesting comparison is the changes in the radius of the charcoal particle during gasification predicted by the model depending on whether fragmentation is taken into account or not. For this purpose, we used the experimental results obtained by Mermoud et al. [6] for a charcoal particle with an initial diameter of $30 \mathrm{~mm}$ which was partially gasified to $20 \%, 50 \%$ and $70 \%$ at $930^{\circ} \mathrm{C}$ and $0.2 \mathrm{~atm} \mathrm{H}_{2} \mathrm{O}$ and recovered for macro- and microscopic observations. The size of the particle was measured at each conversion value. Given the wood anisotropy, the diameter of the particle is represented with an error interval representing the ovoid shape of the particle. Results are presented in Fig. 7 and compared with the predictions made by the two models. There was much better agreement between experimental measurements and model predictions when the fragmentation phenomenon was taken into account. The discrepancy between the two numerical models corresponds to shrinking of the radius due to the peripheral fragmentation process predicted by our theory.

\section{Conclusion}

In this study, we focused on the problem of modelling gasification at high conversion levels for a single large particle of wood charcoal. In fact, none of the current models succeeds in reproducing the quasi-constant conversion rate observed experimentally. In this study, the classically observed discrepancy between model predictions and experiments was assumed to be due to peripheral fragmentation. From the point of view of the percolation theory, this phenomenon can be considered as the breakage of the connections between isolated clusters and the particle surface. The detachment of additional mass could explain the conservation of the conversion rate in spite of the increase in diffusional effects with an increase in particle size. Different approaches were tested to take into account such a phenomenon and model results were compared to experimental results obtained by Mermoud et al. [6]. Two main conclusions can be drawn from this study:

- Peripheral fragmentation is sensitive to mass and heat transfer limitations within the particle and hence to particle size. As a consequence, a fragmentation criterion based only on a critical porosity value cannot explain the behaviour observed experimentally.

- We have demonstrated that adding a criterion based on the porosity gradient at the surface allowed us to significantly improve modelling of charcoal particle gasification. The introduction of such a parameter enables the impact of diffusional effects on fragmentation to be taken into account.

The upscaling of fragmentation processes to the reactor-scale, which represents the ultimate goal of such a work, is a tremendous task and is beyond the scope of this paper. Moreover, additional mechanisms known to induce fragmentation in industrial gasifiers such as particle attrition [33], bed compaction [38] or the pressure increase during devolatization [16] should be also taken into account. The reader interested by the way of implementing fragmentation in reactor modelling and by a state-of-art on the subject may refer to a recent study of Syred et al. [39]. Nevertheless, in order to give a brief insight into possible routes for an extent of this study to the upper scale, two approaches should be considered as a function of the size (and the type) of char particles under consideration, and hence of the kind of reactor:

- For large particles as the ones under study here, the fragments size produced during gasification can be considered as negligible. For reactors involving such particles (e.g., in fixed bed reactors), the implementation could be quite straightforward. The 
fragments can be assumed to be instantaneously consumed and consequently, the fragmentation process can be directly coupled to gasification [25]. A new criterion - including the above-mentioned fragmentation mechanisms - should be defined in that scope.

- If char particles involved in the gasification process are smaller (few millimetres or less), the fragments size may be significant and their presence cannot be neglected anymore. For such reactors (e.g., in fluidized bed reactors), if our criterion allows to predict the char mass lost by fragmentation, it is not sufficient to determine the evolution of the particle size distribution during the char gasification phase. For certain coals or charcoals, indeed, fragmentation can lead to the formation of several hundreds of new particles - of size almost equal to the fragmenting particles [38]. Hence, a statistical model is required in parallel for predicting the number and the size of fragments generated [30-33].

\section{References}

[1] Dasappa S, Paul PJ, Mukunda HS, Shrinivasa U. The gasification of wood-char spheres in $\mathrm{CO}_{2}-\mathrm{N}_{2}$ mixtures-analysis and experiments. Chem Eng Sci 1994;49:223-32.

[2] Dasappa S, Paul PJ, Mukunda HS, Shrinivasa U. Wood-char gasification: experiments and analysis on single particles and packed beds. Proc Combust Inst 1998;27:1335-42.

[3] Gobel B, Henriksen U, Qvale B, Houbak B. In: Bridgwater A, editor. Progress in thermochemical biomass conversion, vol. 92. Oxford: Blackwell; 2001. p. 108.

[4] Peters B, Bruch C. A flexible and stable numerical method for simulating the thermal decomposition of wood particles. Chemosphere 2001;42:481-90.

[5] Wang FY, Bhatia SY. A generalised dynamic model for char particle gasification with structure evolution and peripheral fragmentation. Chem Eng Sci 2001;56:3683-97.

[6] Mermoud F, Golfier F, Salvador S, Van de steene L, Dirion JL. Experimental and numerical study of steam gasification of a single charcoal particle. Combust Flame 2006;145:59-79.

[7] Struis RPWJ, Von Scala C, Stucki S, Prins R. Gasification reactivity of charcoal with $\mathrm{CO}_{2}$. Part I: conversion and structural phenomena. Chem Eng Sci 2002;57:3581-92

[8] Standish N, Tanjung AFA. Gasification of single wood charcoal particles in $\mathrm{CO}_{2}$. Fuel 1988;67:666-72.

[9] Reyes S, Jensen KF. Percolation concepts in modelling of gas-solid reactions II. Application to char gasification in the diffusion regime. Chem Eng Sci 1986;41:345-54.

[10] Marban G, Fuertes AB. Influence of percolation on the modification of overall particle properties during gasification of porous solids. Chem Eng Sci 1997;52:1-11.

[11] Bar-Ziv E, Kantorovich II. Mutual effects of porosity and reactivity in char oxidation. Prog Energy Combust Sci 2001;27:667-97.

[12] Daccord G. Dissolution, evaporations, etchings. In: Avnir D, editor. The fractal approach to heterogeneous chemistry, vol. 183. New York: John Wiley \& Sons Ltd; 1989. p. 197.

[13] Sahimi M, Gavalas GR, Tsotsis TT. Statistical and continuum models of fluid solid reactions in porous media. Chem Eng Sci 1990;45:1443-502.
[14] Kerstein AR, Niksa S. Fragmentation during carbon conversion: predictions and measurements. Proc Combust Inst 1985;20:941-50.

[15] Sundback CA, Beer JM, Sarofim AF. Fragmentation behavior of single coal particle in a fluidized bed. In: Twentieth symposium on combustion. The Combustion Institute, vol. 20; 1985. p. 1495-503.

[16] Mitchell RE, Akanetuk AEJ. The impact of fragmentation on char conversion during pulverized coal combustion. Proc Combust Inst 1996;26:3137-44.

[17] Zhang H, Cen K, Yan J, Ni M. The fragmentation of coal particles during the coal combustion in a fluidized bed. Fuel 2002;81:1835-40.

[18] Feng B, Bhatia SK. Percolative fragmentation of char particles during gasification. Energ Fuel 2000;14:297-307.

[19] Baxter LL. Char fragmentation and fly ash formation during pulverized-coal combustion. Combust Flame 1992;90:174-84.

[20] Fuertes AB, Marban G. Modelling gasification reactions including the percolation phenomenon. Chem Eng Sci 1994;49:3813-21.

[21] Reyes S, Jensen KF. Percolation concepts in modelling of gas-solid reactions - I. Application to char gasification in the kinetic regime. Chem Eng Sci 1986;41:333-43.

[22] Biggs MJ, Agarwal PK. The $\mathrm{CO} / \mathrm{CO}_{2}$ product ratio for a porous char particle within an incipiently fluidized bed: a numerical study. Chem Eng Sci 1997;52:941-52.

[23] Yamashita T, Fujii Y, Morozumi Y, Aoki H, Miura T. Modeling of gasification and fragmentation behavior of char particles having complicated structures. Combust Flame 2006;146:85-94.

[24] Liu G, Wu H, Gupta HP, Lucas JA, Tate AG, Wall TF. Modeling the fragmentation of non-uniform porous char particles during pulverized coal combustion. Fuel 2000;79:627-33.

[25] Zolin A, Jensen A, Dam-Johansen K. Coupling thermal deactivation with oxidation for predicting the combustion of a solid fuel. Combust Flame 2001;125:1341-60.

[26] Marban G, Pis J, Fuertes AB. Simulation of secondary fragmentation during fluidized bed combustion of char particles. Powder Technol 1996;89:71-8.

[27] Pinho C. Fragmentation on batches of coke or char particles during fluidized bed combustion. Chem Eng J 2006;115:147-55.

[28] Kerstein AR, Edwards BF. Percolation model for simulation of char oxidation and fragmentation time-histories. Chem Eng Sci 1987;42:1629-34.

[29] K Sahimi M, Totsis TT. Dynamic scaling for the fragmentation of reactive porous media. Phys Rev Lett 1987;59:888-91.

[30] Chirone R, Salatino P, Massimilla L. Secondary fragmentation of char particles during combustion in a fluidized bed. Combust Flame 1989;77:79-90.

[31] Cui Y, Stubington JF. In-bed char combustion of Australian coals in PFBC. 3. Secondary fragmentation. Fuel 2001;80:2245-51.

[32] Miccio F, Salatino P. Monte-Carlo Simulation of combustion-induced percolative fragmentation of carbons. Proc Combust Inst 1992:24:1145-51.

[33] Salatino P, Miccio F, Massimilla L. Combustion and percolative fragmentation of carbons. Combust Flame 1993;95:342-50.

[34] Gavalas GR. Analysis of char combustion including the effect of pore enlargement. Combust Sci Technol 1981;24:197-210.

[35] Mohanty KK, Ottino JM, Davis HT. Reaction and transport in disordered composite media: introduction of percolation concepts. Chem Eng Sci 1982;37:905-24.

[36] Shah N, Ottino JM. Transport and reaction in evolving;disordered composites. I: gasification of porous solids. Chem Eng Sci 1987;42:63-72.

[37] Sahimi M. Transport, reaction and fragmentation in evolving porous media. Phys Rev A 1991;43:5367-76.

[38] Fernandes NCP, Castro JAAM. Steady-state simulation of a continuous moving bed reactor in the pulp and paper industry. Chem Eng Sci 2000;55:3729-38.

[39] Syred N, Kurniawan K, Griffiths T, Gralton T, Ray R. Development of fragmentation models for solid fuel combustion and gasification as subroutines for inclusion in CFD codes. Fuel 2007;86:2221-31. 